\title{
Sampled fiber grating dispersion compensation technology based swarm optimization algorithm
}

\author{
Zhu Dandan ${ }^{1,2, a}$, Hu lin $^{1,}$,, Shen Haohao ${ }^{1, a}$, Xi Sibo ${ }^{1,}$, \\ ${ }^{1}$ College of Electric Engineering of YanShan university, \\ ${ }^{2}$ Laboratory of Measurement Technology and Instrumentation of Hebei Province, \\ Qinhuangdao,Hebei province, China \\ ae-mail: zhudandan1896@126.com
}

Keywords: Sampled grating, Dispersion compensation, Delay, Chirp coefficient

\begin{abstract}
Reflective spectrum of sampled grating was analyzed using transfer matrix method. Dispersion compensator was designed by selecting the length of sampled grating, sampled period, sampled rate, chirp coefficient and some kind of appropriate parameters. In multi-channel dispersion compensation simulation experiment, the reflective spectrum of sampled grating was even and reflectivity was nearly above $90 \%$. Time delay spectrum has very good consistency and the best result was that delay jitter was less than 40ps. Dispersion diagram was also even, so the positive and negative dispersion can be compensated. Pure phase sampled function was designed using particle swarm optimization algorithm. Highly uniform and low energy band grating was created. Multi channel sampled grating was made in tunable dispersion compensation.
\end{abstract}

\section{Introduction.}

It was a current research focus on compensating every wavelength channel of dispersion in DWDM optical fiber communication system using a single sampled fiber grating. Due to fiber materials dispersion, the different frequency components of light pulse have different group velocity in optical fiber transmission. Therefore it needs different time arriving at the transmission end. Pulse broadening will occur, it makes the time intervals between adjacent signal overlapping. It cause bit error. Therefore, to solve the problem of the dispersion and dispersion slope compensation of optical fiber was the one of the main problem improving the transmission capacity and distance. Introduction of sampled fiber Bragg grating can improve significantly the effective transmission distance and reliability of optical fiber communication system. It can compensate the dispersion of multiple wavelength channels in DWDM system with a single sampled grating. It has the vital significance to the development of the dense wavelength division multiplexing system. Dispersion compensation properties using chirped sampled fiber Bragg grating was studied in the paper. Adjust the length of the chirped sampled fiber Bragg grating, sampled rate, the index modulation, chirp coefficient and some other parameters for the sampled grating dispersion compensation.

\section{The dispersion compensator design}

Sampled fiber Bragg grating structure was shown in Figure. 1. The period of sampled grating was p, length of exposure zone was a, The total length of sampled grating $\mathrm{L}$.

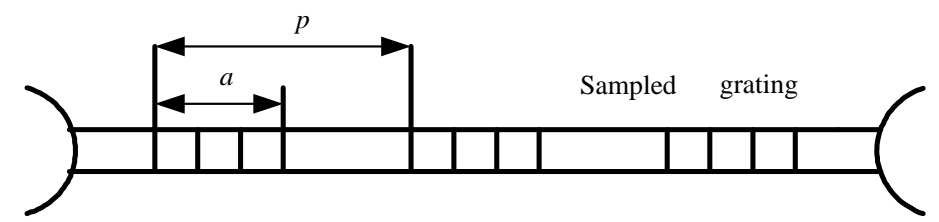

Fig. 1 Schematic diagram of the structure of sampled grating

Sampled grating was analyzed using transfer matrix method in this paper. It can design dispersion compensator by adjusting the chirp coefficient. Chirped sampled fiber Bragg grating 
dispersion compensator design as shown in Figure. 2, sampled grating length $L=1.0345 \mathrm{~cm}$, chirp coefficient $f=9$, the refractive index modulation quantity $\delta n_{\text {eff }}=0.0014$,sampled period was $p=0.65 \mathrm{~mm}$, sampled rate was $R=0.2$. Chirped sampled grating center wavelength were $1548.35 \mathrm{~nm}, 1548.74 \mathrm{~nm}, 1549.14 \mathrm{~nm}, 1549.54 \mathrm{~nm}, 1549.94 \mathrm{~nm}, 1550.34 \mathrm{~nm}, 1.550 .74 \mathrm{~nm}$, $1551.14 \mathrm{~nm}, 1551.54 \mathrm{~nm}, 1.551 .94 \mathrm{~nm}$, channel spacing was $0.4 \mathrm{~nm}$., We can see that reflection spectrum and delay curve of each channel chirped in sampled grating has good consistency from Fig. 2 . The amount of dispersion was relatively uniform, and can reach $1000 \mathrm{ps} / \mathrm{nm}$, delay jitter was less than 40ps.
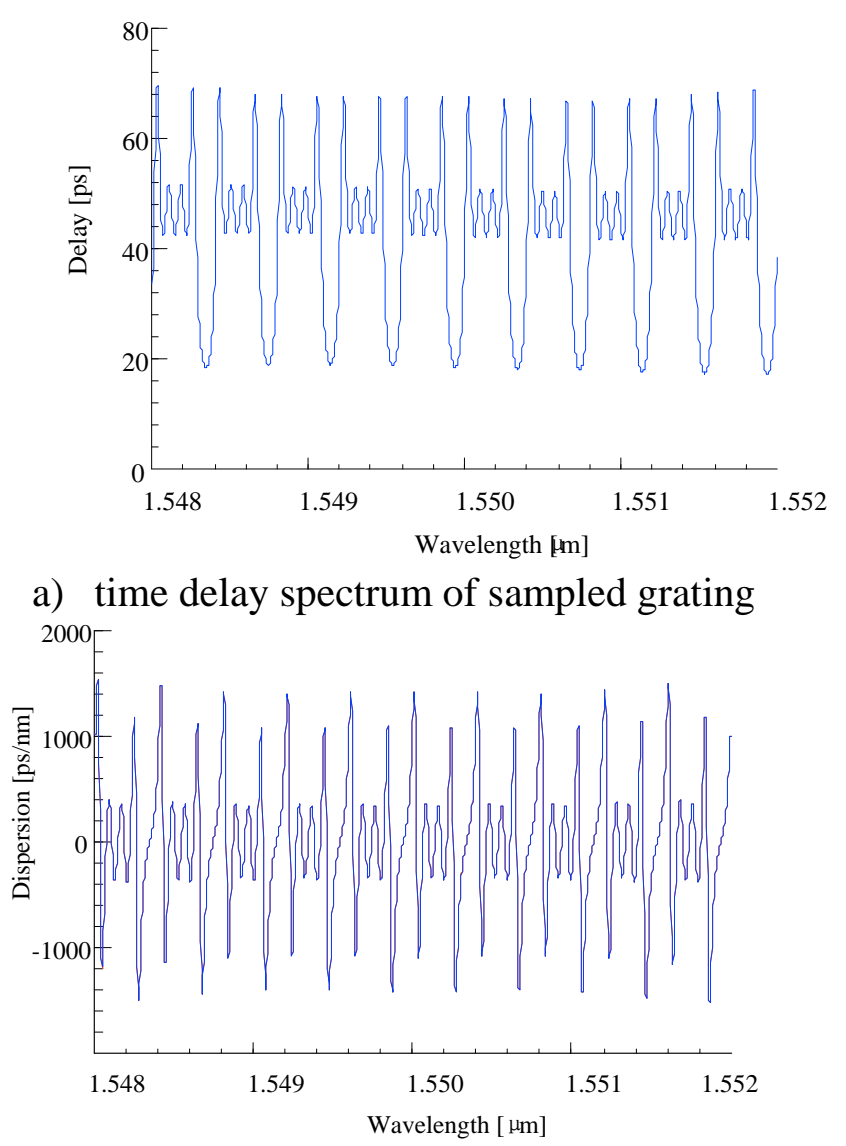

b) dispersion spectrum of sampled grating

Fig.2 Reflective spectrum, time delay spectrum and dispersion spectrum when channel spacing was $0.4 \mathrm{~nm}$

\section{Particle swarm optimization algorithm.}

The refractive index modulation coefficient of the phase sampled fiber grating can be expressed as:

$$
\delta n_{e f f}(z)=\delta \overline{n_{e f f}}(z)\left(1+v \operatorname{Re}\left\{\exp \left[i\left(\frac{2 \pi}{\Lambda} z+\phi(z)\right)\right]\right\}\right.
$$

Where $\delta n_{\text {eff }}$ was space average refractive index change in grating period, $v$ was the fringe visibility of refractive index change, $\Lambda$ was the average grating period, $\phi(z)$ was the chirp of grating.

By Fourier transform, the change of refractive index can be expressed as:

$$
\begin{gathered}
\delta n_{\text {eff }}(z)=\delta \overline{n_{\text {eff }}}(z)\left(1+v \operatorname{Re}\left\{\sum_{m}\left[\frac{1}{M} \sum_{k=0}^{N-1} \exp (i 2 \pi \phi(k)) \exp (-i k 2 \pi m / M)\right] \exp \left[i\left(\frac{2 \pi}{\Lambda} z+\frac{2 \pi}{\Lambda} m\right)\right]\right\}\right) \\
m=0,1,2, \ldots M
\end{gathered}
$$


The peak reflectivity of $\mathrm{m}$ channel phase sampled fiber Bragg grating can be expressed as:

$$
r_{m}=\tanh ^{2}\left(\mid \frac{1}{M} \frac{\pi \delta \overline{n_{e f f}}}{\lambda_{B}} \sum_{m}\left[\frac{1}{M} \sum_{k=0}^{N-1} \exp (i 2 \pi \phi(k)) \exp (-i k 2 \pi m / M)\right] L\right)
$$

Where $L$ was the total length of sampled grating. In order to get the ideal reflectivity of reflective peak, the problem was transformed into minimization problem of formula (4)

$$
C=\frac{1}{M} \sum_{m} W_{m}\left|r_{0}-r_{m}\right|^{2}
$$

C was optimization objective function. $W_{m}$ was the first $\mathrm{M}$ channel reflectivity factor. Its value was between 0 and 1. $r_{0}$ was the target reflectivity. Minimization of the objective function $\mathrm{C}$ can be achieved by optimizing the $\phi(z)$ function. Particle swarm optimization algorithm was selected. The calculation steps were as the following

(1)position and velocity of all the individuals were initialized in the D dimension search space, dimension represents the number of required optimized $\phi(z)$.

(2)the target function values of each particle was calculated under D dimension variable.

(3)The objective function value of each particle compared with its optimal value previous. If the new value was good, then it replaced the old value. The position of optimal value was recorded. If not the value remain unchanged.

(4)optimal objective function value of adjacent to the individual and global individual was compared. If an individual objective function value was better than the current optimal value. It replaced the old value. The position and number of individual was recorded. If not the value remain unchanged.

(5)velocity and position was updated, entering the next cycle until the termination condition was met. Error function was

$$
T=\frac{1}{M} \sum_{m=1}^{M} W_{m}\left(r_{m}-r_{0}\right)^{2}
$$

Where, $\mathrm{M}$ was the total number of reflective orders, $W_{m}$ was the weight of the channel, $r_{m}$ was the size of the reflectance of the reflection peak every level times . $r_{0}$ was determined target of peak reflectivity

\section{Summary}

In this paper, sampled grating was analyzed based on the transfer matrix method and coupled-mode theory. The influence of parameters on sampled grating reflective characteristics were studied. It can get proper reflective law. According to this law, The application characteristics of chirped sampled grating in the dispersion compensation were studied. Chirped fiber grating dispersion compensators was designed. Reflective and dispersion characteristics in each cycle were basically same. Reflective spectrum of sampled grating was even and reflectivity was nearly above $90 \%$. delay jitter is less than 40ps. Sampled grating can be used for dispersion compensation. Phase distribution of pure phase sampled grating were optimized using particle swarm optimization. It can get high channel and good channel uniformity sampled grating. Sampled grating achieve the purpose of dispersion compensation.

\section{Acknowledgments}

It is supported by postdoctoral scientific research project of Hebei Province(B2014003012)and the natural science foundation of Hebei Province (F2014203157) 


\section{References}

[1]C.H.Wang, L.R.Chen, P.W.E. Smith. Analysis of chirped-super structure and sampled-chirped fiber Bragg gratings. Applied Optics. vol.41, no.9, pp. 1654-1660, 2002

[2]ERDOGAN T. Fiber grating spectra[J]. Lightwave Technol. vol.15, no.8, pp. 1277-1294, 1997

[3]Yuzhe Yin, Xiangfei Chen, Lin Zhu. Analytical expression and system simulation of eye opening penalty for chirped super structure Bragg grating dispersion compensator. Optics Communications. vol.20, no.3, pp. 93-99, 2002

[4]G.P. Agrawal, S. Radic. Phase-shifted fiber Bragg gratings and their application for wavelength demultiplexing .IEEE Photonics Technology Lett, vol.6, no.8, pp. 995, 2012

[5]N.F. Güler,I. Navruz, The optical grating based solutions for dispersion compensation in optical communication systems, J. Fac. Eng, vol.21, no.1, pp. 129-136, 2006

[6]A.D. Kersey et al..Dual wavelength fiber interferometer with wavelength selection via fiber Bragg grating elements. Electron. Lett.IEE JNL.1992,28(13):1215-1216 